\title{
Hereditary hypophosphatemic rickets and spontaneous dental abscesses: a case report
}

\author{
T-M Croonenborghs ${ }^{1 *}$, K Grisar ${ }^{1}$ and C Politis ${ }^{1}$ \\ ${ }^{1}$ OMFS-IMPATH research group, Dept. Imaging \& Pathology, Faculty of Medicine, University Leuven and Maxillofacial Surgery Department, University Hospitals \\ Leuven, Leuven, Belgium
}

\begin{abstract}
This case report describes the dental complications and the coinciding diagnostic and therapeutic challenges in a 25 -year old male with vitamin D-resistant hereditary rickets. Our approach includes prevention by improvement of dental care, initial conservative treatment using antibiotics and subsequent relapse was treated with a combined surgical and endodontic approach.
\end{abstract}

\section{Background}

Vitamin D-resistant hypophosphatemic rickets, also known as familial or hereditary hypophosphatemic rickets (HHR), results in renal phosphate wasting and has a global prevalence estimate of 1:20.000 $[1,2]$. Two causes need to be considered in renal phosphate wasting: on the one hand caused by a primary renal tubular defect, which won't be further discussed, on the other hand as a secondary effect due to increased fibroblast growth factor 23 (FGF23) signaling, as is seen in HHR [3]. The increased FGF-23 has a dual effect in HHR. Firstly, it inhibits renal phosphate resorption in the proximal tubule of the nephron by decreasing the number of sodium-transporters. Secondly FGF-23 also inhibits 1-a-hydroxylase, which diminishes vitamin D activation. Consequently, renal phosphate reabsorption and intestinal uptake of phosphate and calcium will decrease respectively [4].

Current literature describes four genetic mutations resulting in secondary HHR. Firstly and most frequently, a mutation on the $\mathrm{X}$-linked PHEX gene (Phosphate-regulating gene with homologies to endopeptidases on the X-chromosome) is identified. The other identified hereditary etiologies are: autosomal recessive hypophosphatemic rickets (ARHR) and autosomal dominant hypophosphatemic rickets (ADHR), with respectively DMP1 (dentin matrix protein 1; ARHR1), ENPP1 (Ectonucleotide pyrophosphatase/phosphodiesterase 1; ARHR2) and FGF23 (ADHR) affected by mutations $[2,3,5,6]$.

Clinical signs include skeletal aberrancies: short stature, (lower limb) bone deformities and frontal bossing, as well as muscular complaints and dental anomalies. The latter results in spontaneous periapical abscesses and fistulae occurring without any history of trauma or dental decay [7]. The symptomatology can vary based on several factors, such as family history, the extent of hypophosphatemia and current age. The importance of age is twofold: the age of systemic therapy onset is important, but moreover, age determines the current phase of dentition, given that deciduous teeth are mostly more affected compared to permanent teeth $[2,8,9]$. Radiology will display teeth with short roots, large pulp chambers, high pulp horns and sometimes taurodontism. Some of these alterations can persist to a lesser extent in early-treated, compliant patients $[8,9]$.
Histological analysis points out that the dysplastic dentin exhibits large tubular clefts or lacunae reaching the dentino-enamel junction. Because of the lack of calcospherite fusion, large interglobular spaces are formed which are filled with non-mineralized matrix. The mantle dentin is, in contrast to the circumpulpal dentin, usually not or less affected in early treated patients $[2,8,9]$. A micro CT-study by Ribeiro et al. endorses these findings [10]. Furthermore, displaying large pulp chambers with an abnormally high pulp/tooth volume ratio $[7,11]$. Regarding structural deformities of the enamel current evidence is conflicting $[2,7,8]$. The standing hypothesis states that these structural dental deformities facilitate the progression of bacteria to the pulp, leading to infection of the pulp chamber $[2,8]$. Moreover, recent research indicates that subjects with HHR are prone to periodontitis and periodontal bone loss, presumably due to hypoplastic cementum $[12,13]$.

This case report concerning an adult with HHR exposes the need for a systemic and intra-oral treatment strategy, for patients presenting with dental complications in adulthood. Furthermore, conforming the importance of early diagnosis and start of therapy.

\section{Case presentation}

A 25-year-old Caucasian male was referred to the department of Oral and Maxillofacial Surgery by his dental practitioner because of persisting dental complaints since a few weeks, including episodes of a throbbing pain. Antibiotics, Amoxicillin 1 gram twice a day, were prescribed by his dentist 5 days ago.

*Correspondence to: Tomas-Marijn Croonenborghs, OMFS-IMPATH research group, Dept. Imaging \& Pathology, Faculty of Medicine, University Leuven and Maxillofacial Surgery Department, University Hospitals Leuven, Kapucijnenvoer 33, 3000 Leuven, Belgium, Tel: 003216332480; E-mail: tomasmarijn.croonenborghs@uzleuven.be

Key words: Hereditary rickets, Hypophosphatemia, Dental abscess

Received: March 12, 2018; Accepted: March 28, 2018; Published: March 30, 2018 
The medical history of the patient revealed HHR disease, diagnosed at the age of 2 at our hospital. The initiated treatment included phosphate and vitamin D substitution, combined with regular clinical follow up. A hereditary transmission of the disease is suspected, given that his sister and their mother are also affected, although the latter only to a lesser degree (Figure 1). In 2008, genetic screening for mutations on the PHEX-, FGF23- \& DMP1-genes was performed, but no causal DNA aberrancy was identified. The disease elicited multiple complications: a partial craniosynostosis, arterial hypertension, nephrocalcinosis and presumably tertiary hyperparathyroidism. Surgically, a bilateral Ilizarov deformity correction and a total parathyroidectomy were performed. In addition, several dental antecedents were identified: multiple decayed deciduous elements were extracted, protection of the molars was provided by application of a sealant resin and 4 premolars were extracted as a result of crowding. The upper left central incisor, lower left canine and primary molar (21-33-36) had undergone endodontic treatment in the past, in the case of the incisor combined with crown replacement because of dental trauma. Current daily medications include Amoxicilline 1 gram twice a day, phosphate and calcium supplementation (respectively 1200 and $1250 \mathrm{mg}$ ), Amlor 10 mg and 1-Alfa-Leo 1 ggr 5 days a week. The patient has no identified allergies, he is a non-smoker and occasionally consumes alcohol.

General clinical examinations displayed a rather short stature and frontal bossing. Intra-oral inspection revealed a poor general oral hygiene, with percussion pain at the lower left premolar (34) and second molar (37), but no pain sensation at the lower right second molar (47) (Figure 2a and 2b). Temperature and electric vitality tests elicited an adequate response at the lower right and left secondary molars (37-47). Radiographic examinations, including a panoramic view and cone beam CT-scan, showed periapical translucencies at the upper left central incisor, the lower left canine, the lower left primary and secondary molars and the lower right secondary molar (21-33-3637-47) (Figure 2c). Moreover, it displayed enlarged pulp chambers and pulp horns, nearby the dentino-enamel junction.

Antibiotics were continued and further diagnostic work up was completed with a blood sample, bone scintigraphy and lower left quadrant apical biopsies. The blood sample analyzing the current calcium- and phosphate status, showed a slightly lowered phosphate $0,76 \mathrm{mmol} / \mathrm{L}$ (lower limit of normal (LLN): $0,81 \mathrm{mmol} / \mathrm{L}$ ), alkaline phosphatase $39 \mathrm{U} / \mathrm{L}$ (LLN: 40U/L) and parathyroid hormone 11,8 ng/L (LLN: 14,9 ng/L). A combined bone scintigraphy-CT-scan identified bilateral mandibular hypercaptation and a humeral osteolytic lesion on the left side (Figure 3). An MRI was performed and the lesion turned out to be a simple bone cyst. Apical mandibular biopsies were subsequently taken at the lower left canine, primary and secondary left molars and the lower right secondary molar (33-36-37-47), but solely identified inflammatory tissue (Figure 4).

Because of clinical improvement and comforting test results, there was a multidisciplinary preference for follow up concerning the dental complaints as well as the simple humeral bone cyst. However, 6 months after the first visit, the patient returned with similar dental complaints. Clinical investigation showed fluctuation in the apical region of the lower left primary molar (36) and spontaneous pus evacuation apical at the lower left secondary molar (37). Radiographic examinations revealed an increase in apical radiolucency at the left primary molar and both lower right and left secondary molars (36-37-47) (Figure 5). Endodontic treatment with apex resection of mesial roots of the lower left primary and secondary molars (36-37) was performed (Figure 6). The lower right secondary molar showed radiographic signs of

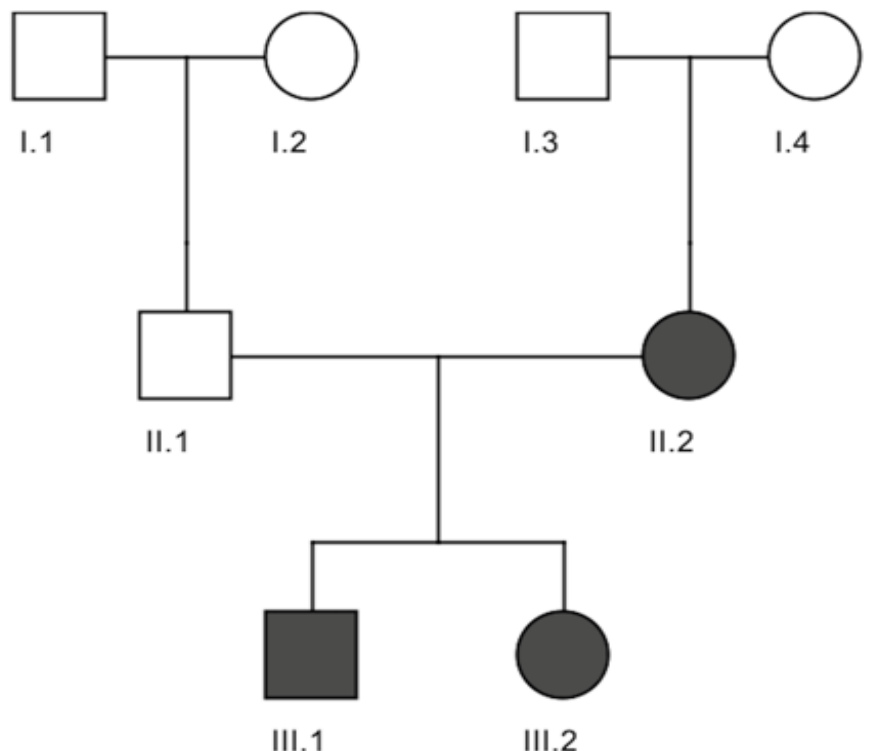

Figure 1. Genogram of the family. Square indicates male, circle indicates female and solid means affected
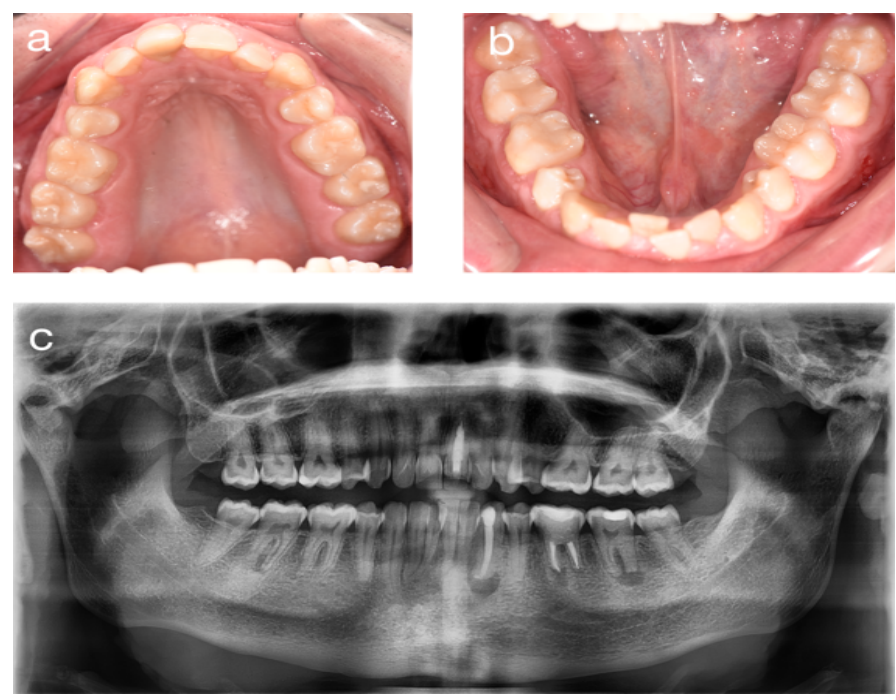

Figure 2. Initial status of the patient. ( $a$ and $b$ ) Intraoral view of maxilla and mandible showing signs of dental crowding, but no apparent signs of caries. (c) Panoramic view from the first consultation

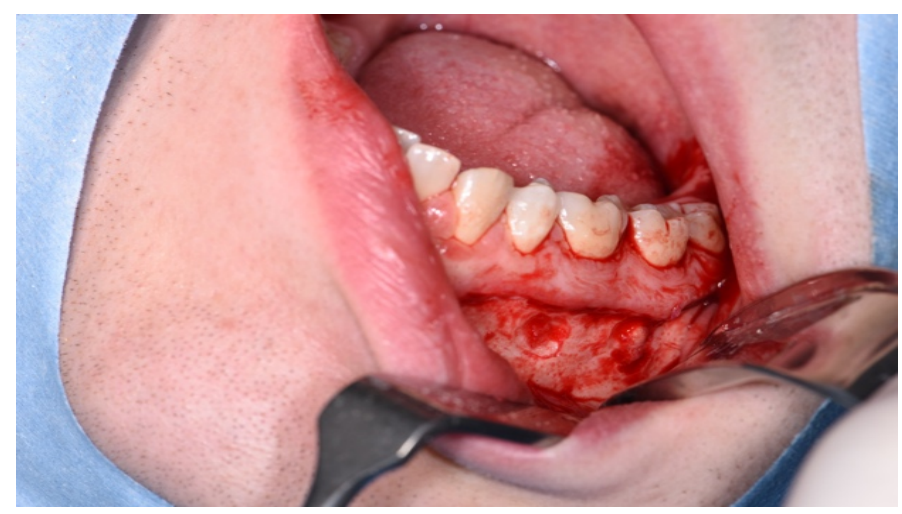

Figure 3. Perioperative clinical photograph. Taken during the apical biopsy procedure in the lower left quadrant 


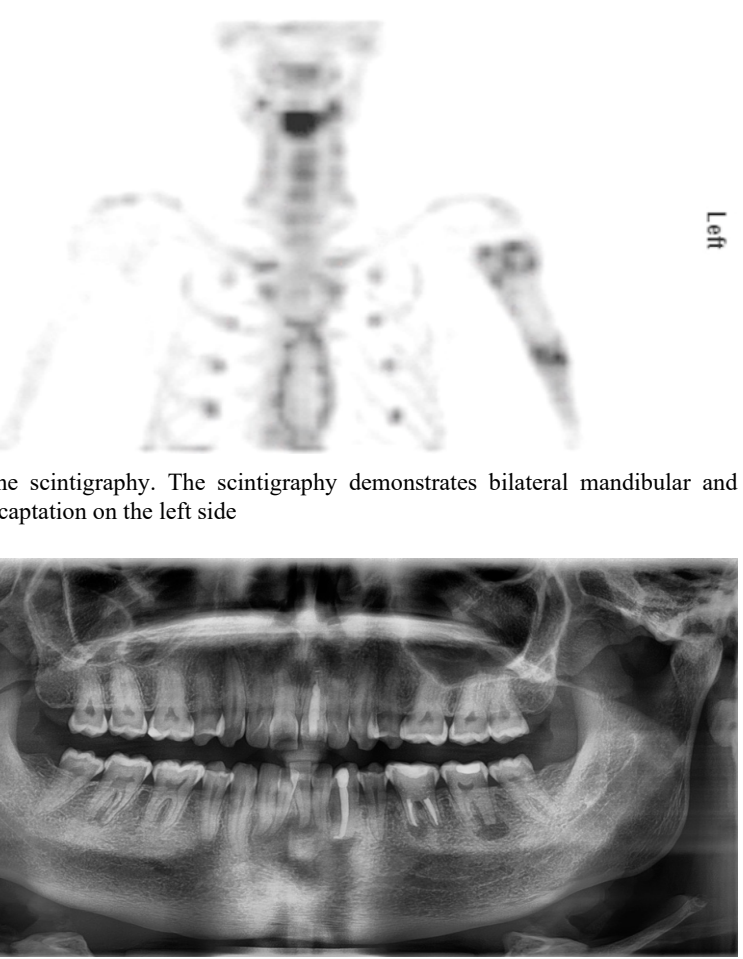

Figure 5. Preoperative panoramic view
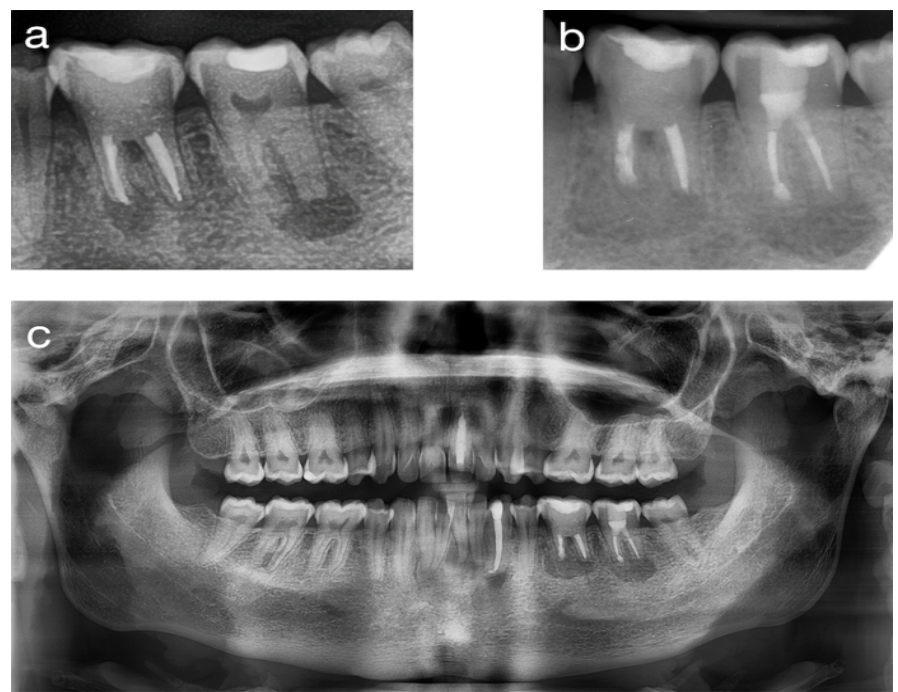

Figure 6. Pre- and postoperative radiographic comparison. (a) Preoperative radiograph of the lower left quadrant. (b) Postoperative apical radiograph of the lower left quadrant. (c) Postoperative panoramic view

progression, however the lesion was complaint-free, deeming treatment unnecessary. Postoperatively, the patient complained of hypoesthesia in the left nervus mentalis region. Befact Forte was started to potentiate nerve recuperation, achieving full sensory recovery 6 months later.

\section{Discussion}

Multiple abscesses and fistulae, typically without dental decay, are common complications in patients with HHR [7,9]. As was demonstrated in the case, enlarged pulp chambers and high pulp horns are identified to a lesser extent in early and adequately treated patients $[7,8,11,12]$. The absolute number of affected teeth rises with age and the distribution pattern changes from mesial to distal $[14,15]$. Most of the current research in hereditary rickets is focused on child population, as such more longitudinal studies assessing the influence of aging, attrition and abrasion on the affected dentin and enamel are desirable.

Recent studies concerning the periodontal status in a HHR population, revealed evidence of increased susceptibility for periodontitis. Periodontal disease is more prevalent and severe when therapy compliancy was moderate during childhood. Moreover, a nonsignificant trend indicated better periodontal health when substitution herapy is continued during adulthood $[12,13]$. The influence of the secondary and tertiary hyperparathyroidism on the mentioned oral complications and anomalies is currently unknown.

A DNA-mutation is the likely suspect as the cause of HHR, since a familial inheritance pattern is identified (Figure 1). Most plausible are $\mathrm{X}$-linked or autosomal dominant patterns of inheritance. The autosomal recessive pattern was deemed improbable, hence consanguinity was excluded [13]. Nevertheless, no causative genetic mutation was ascertained on the PHEX-gene, nor on the DMP1- or FGF23-genes. However, the reasons why these results should be interpreted with the necessary caution are twofold. Firstly, the DNA was sequenced for the 3 genes, performing PCR only on the PHEX-gene. Thus no sensitivity augmenting tests like denaturing high-performance liquid chromatography (dHPLC), nor multiplex ligation-dependent probe amplification analysis (MPLA) were used $[6,16]$. Secondly, however improbable, the autosomal recessive ENPP1 mutation was not yet identified as a causative mutation at that time [6].

Combining HHR with poor oral hygiene increases the risk for dental complications significantly [9]. Hence demonstrating the persistent importance of good oral hygiene in adulthood. When confronted with dental abscesses in patients with HHR, endodontic treatment serves as first choice option, possibly combined with an apex resection $[17,18]$. Antibiotic treatment isn't mentioned in current literature as a valid treatment option in primary infections. Evidently antibiotics won't mend the point of entry for microorganisms, however current evidence indicates that endodontically treated teeth are still at risk for infection in patients with HHR $[1,14]$. So, antibiotics can postpone or possibly prevent the need for invasive treatment in primary infections, as was demonstrated in our case. Besides dental care, systemic therapy serves a vital role in treating HHR. When patients are diagnosed with HHR in their childhood, a sharp-cut treatment program is initiated, including supplementation and specialist follow up. However, concerning treatment of HHR in adulthood there is no treatment strategy, current empirical approach encompasses reinitiating (and maintaining) supplementation in symptomatic patients [3]. Further research concerning intra-oral and systemic therapy in adults with dental complications as a result of HHR, seems a necessity in improving treatment options.

\section{Conclusion}

Early diagnosis and start of systemic and intra-oral treatment, combined with frequent follow up for patients with hereditary rickets, is beneficial in the prevention of dental anomalies and complications. However, maintaining this mantra is problematic. Mostly because a consensus (preventive) treatment strategy during adulthood is currently lacking, for a dentition remaining prone to the invasion of microorganisms. Moreover, besides pediatricians and dentists, general practitioners and internists monitoring patients with HHR, should be aware of the distinct general and dental features. 


\section{Declarations}

Ethics approval and consent to participate: Not applicable.

Consent for publication: Consent for publication was given by the patient.

\section{Availability of data and material: Not applicable.}

Competing interests: All authors approbate to the article and declare to have no conflicts of interest.

Funding: There was no external funding for this work.

Authors' contributions: All authors contributed equally in the design, acquisition of data, drafting of the article and the following revisions. They all read and approved the final manuscript.

\section{Acknowledgements}

We would like to thank Ms. Fien Vantomme for the essential revision and editing of our article.

\section{Conflicts of interest}

The authors report no conflicts of interest related to this study.

\section{References}

1. Souza AP, Kobayashi TY, Lourenço Neto N, Silva SMB, Machado MA, et al. (2013) Dental manifestations of patient with vitamin D-resistant rickets. J Appl Oral Sci 21: 601-606. [Crossref]

2. Douyere D, Joseph C, Gaucher C, Chaussain C, Courson F, et al. (2009) Familial hypophosphatemic vitamin D-resistant rickets-prevention of spontaneous dental abscesses on primary teeth: A case report. Oral Surg Oral Med Oral Pathol Oral Radiol Endod 107: 525-530. [Crossref]

3. Linglart A, Biosse-Duplan M, Briot K, Chaussain C, Esterle L, et al. (2014) Therapeutic management of hypophosphatemic rickets from infancy to adulthood. Endocr Connect 3: R13-30. [Crossref]

4. Souza MA, Valente Soares Junior LA, Dos Santos MA, Vaisbich MH (2010) Dental abnormalities and oral health in patients with Hypophosphatemic rickets. Clinics (Sao Paulo) 65: 1023-1026. [Crossref]

5. Christofori G, Naik P, Douglas H (1995) The hyp consortium. Nat Genet 10: 196-201.
6. Beck-Nielsen SS, Brixen K, Gram J, Brusgaard K (2012) Mutational analysis of PHEX, FGD23, DMP1, SLC34A3 and CLCN5 in patients with hypophosphatemic rickets. $J$ Hum Genet 57:453-458. [Crossref]

7. Baroncelli GI, Angiolini M, Ninni E, Galli V, Saggese R, et al. (2006) Prevalence and pathogenesis of dental and periodontal lesions in children with X-linked hypophosphatemic rickets. Eur J Paediatr Dent 7: 61-66. [Crossref]

8. Cremonesi I, Nucci C, D’Alessandro G, Alkhamis N, Marchionni S, et al. (2014) $\mathrm{X}$-linked hypophosphatemic rickets: Enamel abnormalities and oral clinical findings. Scanning 36: 456-461. [Crossref]

9. Chaussain-Miller C, Sinding C, Wolikow M, Lasfargues JJ, Godeau G, et al. (2003) Dental abnormalities in patients with familial hypophosphatemic vitamin D-resistant rickets: Prevention by early treatment with 1-hydroxyvitamin D. J Pediatr 142: 324331. [Crossref]]

10. Ribeiro T, Costa F, Soares E, Williams J FC (2015) Enamel and dentin mineralization in familial hypophosphatemic rickets?: a micro-CT study. Dentomaxillofacial Radiol 44. [Crossref]

11. Chaussain-Miller C, Sinding C, Septier D, Wolikow M, Goldberg M, et al. (2007) Dentin structure in familial hypophosphatemic rickets: Benefits of vitamin D and phosphate treatment. Oral Dis 13: 482-489. [Crossref]

12. Biosse-Duplan M, Coyac BR, Bardet C, Zadikian C, Rothenbuhler A, et al. (2017) Phosphate and Vitamin D Prevent Periodontitis in X-Linked Hypophosphatemia. $J$ Dent Res 96: 388-395. [Crossref]

13. Ye L, Liu R, White N, Alon US, Cobb CM (2011) Periodontal status of patients with hypophosphatemic rickets: a case series. J Periodontol 82: 1530-1535. [Crossref]

14. Andersen MG, Beck-Nielsen SS, Haubek D, Hintze H, Gjørup H, et al. (2012) Periapical and endodontic status of permanent teeth in patients with hypophosphatemic rickets. J Oral Rehabil 39: 144-150.

15. Beck-Nielsen SS, Brusgaard K, Rasmussen LM, Brixen K, Brock-Jacobsen B, et al. (2010) Phenotype presentation of hypophosphatemic rickets in adults. Calcif Tissue Int 87: 108-119. [Crossref]

16. Stuppia L, Antonucci I, Palka G, Gatta V (2012) Use of the MLPA assay in the molecular diagnosis of gene copy number alterations in human genetic diseases. Int $J$ Mol Sci 13: 3245-3276. [Crossref]

17. Beltes C, Zachou E (2012) Endodontic management in a patient with vitamin D-resistant rickets. $J$ Endod 38: 255-258. [Crossref]

18. Lee BN, Jung HY, Chang HS, Hwang YC, Oh WM (2017) Dental management of patients with X-linked hypophosphatemia. Restor Dent Endod 42: 146-151. [Crossref]

Copyright: $\odot 2018$ Croonenborghs TM. This is an open-access article distributed under the terms of the Creative Commons Attribution License, which permits unrestricted use, distribution, and reproduction in any medium, provided the original author and source are credited. 